\title{
Commutator methods for unitary operators
}

\author{
Claudio Fernández, ${ }^{1}$ Serge Richard, ${ }^{2}$ and Rafael Tiedra de Aldecoa ${ }^{3}$
}

\begin{abstract}
We present an improved version of commutator methods for unitary operators under a weak regularity condition. Once applied to a unitary operator, the method typically leads to the absence of singularly continuous spectrum and to the local finiteness of point spectrum. Large families of locally smooth operators are also exhibited. Half of the paper is dedicated to applications, and a special emphasis is put on the study of cocycles over irrational rotations. It is apparently the first time that commutator methods are applied in the context of rotation algebras, for the study of their generators.
\end{abstract}

Mathematics Subject Classification (2010). 81Q10, 47A35, 47B47.

Keywords. Unitary operators, spectral analysis, Mourre theory, limiting absorption principle, cocycles over rotations.

\section{Introduction}

It is commonly accepted that commutator methods (in the sense of E. Mourre) are very efficient tools for studying spectral properties of self-adjoint operators. These techniques are well developed and have been formulated with a high degree of optimality in [3]. On the other hand, corresponding approaches for the study of unitary operators exist but are much less numerous and still at a preliminary stage of development (see for example [4], [16], [20], [21], and references therein). Accordingly, the aim of this paper is to extend commutator methods for unitary operators up to an optimality equivalent to the one reached for self-adjoint operators, and to present some applications.

Up to our knowledge, it is Putnam who presented the first application of commutator methods for unitary operators. His original result [20], Theorem 2.3.2, reads as

\footnotetext{
${ }^{1}$ Supported by the Fondecyt Grant 1100304, Anillo ACT 1112, and by the ECOS/CONICYT Grant C10E01.

${ }^{2}$ Supported by the Japan Society for the Promotion of Science (JSPS) and by "Grants-in-Aid for scientific Research".

${ }^{3}$ Supported by the Fondecyt Grant 1090008 , by the Iniciativa Cientifica Milenio ICM P07-027-F "Mathematical Theory of Quantum and Classical Magnetic Systems" and by the ECOS/CONICYT Grant C10E01.
} 
follows: if $U$ is a unitary operator in a Hilbert space $\mathscr{H}$ and if there exists a bounded self-adjoint operator $A$ in $\mathscr{H}$ such that $U^{*} A U-A$ is strictly positive, then the spectrum of $U$ is purely absolutely continuous. An extension of this statement to operators $A$ which are only semi-bounded is also presented in that reference. However, in applications even this assumption of semi-boundedness is often too restrictive, and a first attempt to extend the theory without this condition has been proposed in [4]. To do this, the authors had to impose some regularity of $U$ with respect to $A$. In the language of Mourre theory for self-adjoint operators, this regularity assumption corresponds to the inclusion $U \in C^{2}(A)$. Apart from the removal of the semi-boundedness assumption, the applicability of the theory was also significantly broadened by requiring a stronger positivity condition, but only locally in the spectrum of $U$ and up to a compact term. Under these assumptions, the spectrum of $U$ has been shown to consist of an absolute continuous part together with a possible finite set of eigenvalues (see [4], Theorem 3.3, for details).

The main goal of the present work is to weaken the regularity assumption of $U$ with respect to $A$ to get a theory as optimal as the one in the self-adjoint case. Accordingly, we show that all the results on the spectrum of $U$ hold if a condition only slightly stronger than $U \in C^{1}(A)$ is imposed; namely, either if $U \in C^{1,1}(A)$ and $U$ has a spectral gap, or if $U \in C^{1+0}(A)$ (see Section 2.1 and Theorem 2.7 for notations and details). In addition to that result, we also obtain a broader class of locally $U$-smooth operators, and thus more general limiting absorption principles for $U$ (see Proposition 2.9). Our proofs are rather short and natural, thanks to an extensive use of the Cayley transform.

The second half of the paper is dedicated to applications. In Sections 3.1-3.2, we consider perturbations of bilateral shifts as well as perturbations of the Schrödinger free evolution. In both cases, we show that the regularity assumption can be weakened down to the condition $U \in C^{1+0}(A)$. This extends significantly some previous results of [4] in similar situations. Note that any result on the perturbations of the Schrödinger free evolution is of independent interest since it provides information on the corresponding Floquet operators (see [4], [12], and [13] for more details on this issue).

In Section 3.3, we determine spectral properties of cocycles over irrational rotations. It is apparently the first time that commutator methods are applied in the context of rotation algebras, for the study of their generators. For a class of cocycles inspired by [15], and [17], we show that the corresponding unitary operators have purely Lebesgue spectrum. The result is not new [14] but our proof is completely new and does not rely on the study of the Fourier coefficients of the spectral measure. In addition, our approach leads naturally to a limiting absorption principle and to the obtention of a large class of locally smooth operators. This information could certainly not be deduced from the study of the Fourier coefficients alone.

Finally, we treat in Section 3.4 vector fields on orientable manifolds. Under suitable assumptions, we show that the unitary operators induced by a general class of complete vector fields are purely absolutely continuous. This result comple- 
ments [20], Section 2.9(ii), where the author considers the case of unitary operators induced by divergence-free vector fields on connected open subsets of $\mathbb{R}^{n}$.

As a conclusion, let us stress that since unitary operators are bounded, the abstract theory developed below is slightly simpler than its self-adjoint counterpart. This feature, together with the fact that various dynamical systems are described by unitary operators, suggests that the abstract results obtained so far could be applied in a wide class of situations. For example, it would certainly be of interest to see if spectral properties of CMV matrices [9] and [23], quantum kicked rotors [5], [10], quantized Henon maps [7], [26], [27], or integral transforms [8] and [24] can be studied with commutator methods.

Acknowledgements. Claudio Fernández and Rafael Tiedra de Aldecoa thank Olivier Bourget for many useful discussions on the spectral theory for unitary operators. Serge Richard is grateful for the hospitality provided by the Mathematics Department of the Pontificia Universidad Católica de Chile in November 2011. Finally, the authors thank the anonymous referee for helpful advises which led to a drastic improvement of Section 2.

\section{Commutator methods for unitary operators}

2.1. Regularity with respect to $A$. We first recall some definitions and results borrowed from [3]. Let $\mathscr{H}$ be a Hilbert space with norm $\|\cdot\|$ and scalar product $\langle\cdot, \cdot\rangle$, and denote by $\mathcal{B}(\mathscr{H})$ (resp. $\mathcal{K}(\mathscr{H}))$ the set of bounded (resp. compact) linear operators in $\mathscr{H}$. Let $S \in \mathscr{B}(\mathscr{H})$ and let $A$ be a self-adjoint operator in $\mathscr{H}$ with domain $\mathscr{D}(A)$. For any $k \in \mathbb{N}$, we say that $S$ belongs to $C^{k}(A)$, with notation $S \in C^{k}(A)$, if the map

$$
\mathbb{R} \ni t \longmapsto \mathrm{e}^{-i t A} S \mathrm{e}^{i t A} \in \mathscr{B}(\mathscr{H})
$$

is strongly of class $C^{k}$. In the case $k=1$, one has $S \in C^{1}(A)$ if and only if the quadratic form

$$
\mathscr{D}(A) \ni \varphi \longmapsto\langle A \varphi, S \varphi\rangle-\langle\varphi, S A \varphi\rangle \in \mathbb{C}
$$

is continuous for the topology induced by $\mathscr{H}$ on $\mathscr{D}(A)$. The operator corresponding to the continuous extension of the form is denoted by $[A, S] \in \mathscr{B}(\mathscr{H})$ and verifies $[A, S]=\mathrm{s}-\lim _{\tau \rightarrow 0}\left[A_{\tau}, S\right]$ with $A_{\tau} \stackrel{\text { def }}{=}(i \tau)^{-1}\left(\mathrm{e}^{i \tau A}-1\right) \in \mathscr{B}(\mathscr{H})$.

Two slightly stronger regularity conditions than $S \in C^{1}(A)$ are provided by the following definitions: $S$ belongs to $C^{1,1}(A)$, with notation $S \in C^{1,1}(A)$, if

$$
\int_{0}^{1} \frac{\mathrm{d} t}{t^{2}}\left\|\mathrm{e}^{-i t A} S \mathrm{e}^{i t A}+\mathrm{e}^{i t A} S \mathrm{e}^{-i t A}-2 S\right\|<\infty
$$

and $S$ belongs to $C^{1+0}(A)$, with notation $S \in C^{1+0}(A)$, if $S \in C^{1}(A)$ and

$$
\int_{0}^{1} \frac{\mathrm{d} t}{t}\left\|\mathrm{e}^{-i t A}[A, S] \mathrm{e}^{i t A}-[A, S]\right\|<\infty .
$$


If we regard $C^{1}(A), C^{1,1}(A), C^{1+0}(A)$, and $C^{2}(A)$ as subspaces of $\mathscr{B}(\mathscr{H})$, then we have the following inclusions $C^{2}(A) \subset C^{1+0}(A) \subset C^{1,1}(A) \subset C^{1}(A)$. Now, if $H$ is a self-adjoint operator in $\mathscr{H}$ with domain $\mathscr{D}(H)$, we say that $H$ is of class $C^{k}(A)$ (respectively $C^{1,1}(A)$ or $\left.C^{1+0}(A)\right)$ if $(H+i)^{-1} \in C^{k}(A)$ (respectively $(H+i)^{-1} \in C^{1,1}(A)$ or $\left.(H+i)^{-1} \in C^{1+0}(A)\right)$. So, $H$ is of class $C^{1}(A)$ if and only if the quadratic form

$$
\mathscr{D}(A) \ni \varphi \mapsto\left\langle A \varphi,(H+i)^{-1} \varphi\right\rangle-\left\langle\varphi,(H+i)^{-1} A \varphi\right\rangle \in \mathbb{C}
$$

is continuous with the topology induced by $\mathscr{H}$. In such a situation, the set $\mathscr{D}(H) \cap$ $\mathscr{D}(A)$ is a core for $H$ and the quadratic form

$$
\mathscr{D}(H) \cap \mathscr{D}(A) \ni \varphi \longmapsto\langle H \varphi, A \varphi\rangle-\langle A \varphi, H \varphi\rangle
$$

is continuous in the topology of $\mathscr{D}(H)$; see [3], Theorem 6.2.10(b). This form extends then uniquely to a continuous quadratic form on $\mathscr{D}(H)$ which can be identified with a continuous operator $[H, A]$ from $\mathscr{D}(H)$ to the adjoint space $\mathscr{D}(H)^{*}$. Finally, the following relation holds in $\mathscr{B}(\mathscr{H})$ :

$$
\left[A,(H+i)^{-1}\right]=(H+i)^{-1}[H, A](H+i)^{-1} .
$$

2.2. Cayley transform. We collect in this section some results on the relation between a unitary operator and its Cayley transform. For this, we consider a unitary operator $U$ in $\mathscr{H}$ with spectrum $\sigma(U) \subset \mathbb{T} \equiv\{\theta \in \mathbb{C}|| \theta \mid=1\}$, and we first assume that 1 does not belong to the point spectrum $\sigma_{\mathrm{p}}(U)$ of $U$. Then, the range $\operatorname{Ran}(1-U)$ of $1-U$ is dense in $\mathscr{H}$, and the operator

$$
H \varphi \stackrel{\text { def }}{=} i(1+U)(1-U)^{-1} \varphi, \quad \varphi \in \mathscr{D}(H) \stackrel{\text { def }}{=} \operatorname{Ran}(1-U),
$$

is self-adjoint due to a standard result on the Cayley transform [28], Theorem 8.4(b). Moreover, $H$ is bounded if and only if $1 \notin \sigma(U)$, and $H$ satisfies the useful equation

$$
(H+i)^{-1}=\frac{1}{2 i}(1-U) .
$$

Finally, one has the following relations between the spectra $\sigma(H)$ of $H$ and $\sigma(U)$ of $U$. First, $\theta \in \sigma(U)$ if and only if $i \frac{1+\theta}{1-\theta} \in \sigma(H)$, and $\lambda \in \sigma(H)$ if and only if $\frac{\lambda-i}{\lambda+i} \in \sigma(U)$ (in particular, the point $\theta=1$ corresponds, as in a stereographic projection with origin 1 , to the points $\lambda= \pm \infty$ ). Second, if $E^{U}(\cdot)$ denotes the (complex) spectral measure of $U$, i.e. $U=\int_{\mathbb{T}} \theta E^{U}(\mathrm{~d} \theta)$, and if $E^{H}(\cdot)$ denotes the (real) spectral measure of $H$, i.e. $H=\int_{\mathbb{R}} \lambda E^{H}(\mathrm{~d} \lambda)$, then one has for any Borel set $I \subset \mathbb{R}$ the equality

$$
E^{H}(I)=E^{U}(\Theta) \quad \text { with } \Theta \stackrel{\text { def }}{=}\left\{\frac{\lambda-i}{\lambda+i} \mid \lambda \in I\right\} .
$$


Conversely, one has for any Borel set $\Theta \subset \mathbb{\mathbb { T }}$ the equality

$$
E^{U}(\Theta)=E^{H}(I) \quad \text { with } I \stackrel{\text { def }}{=}\left\{\frac{1+\theta}{1-\theta} \mid \theta \in \Theta\right\} .
$$

In the next lemma, we prove some simple but useful relations linking $U$ and $H$.

Lemma 2.1. Let $U$ and $A$ be respectively a unitary and a self-adjoint operator in $\mathscr{H}$, with $1 \notin \sigma_{\mathrm{p}}(U)$. Let $H$ be the self-adjoint operator defined by (2.2). Then,

(a) $H$ is of class $C^{1}(A)$ if and only if $U \in C^{1}(A)$, in which case

$$
\left[A,(H+i)^{-1}\right]=\frac{i}{2}[A, U]
$$

in $\mathscr{B}(\mathscr{H})$ and

$$
[i H, A]=-2\left\{(1-U)^{-1}\right\}^{*} U^{*}[A, U](1-U)^{-1}
$$

in $\mathscr{B}\left(\mathscr{D}(H), \mathscr{D}(H)^{*}\right)$;

(b) $H$ is of class $C^{1,1}(A)$ (resp. $C^{1+0}(A)$ ) if and only if $U \in C^{1,1}(A)$ (resp. $\left.U \in C^{1+0}(A)\right)$.

Proof. By taking relation (2.3) into account, one gets for any $\varphi \in \mathscr{D}(A)$

$$
\begin{aligned}
\langle A \varphi, & \left.(H+i)^{-1} \varphi\right\rangle-\left\langle\varphi,(H+i)^{-1} A \varphi\right\rangle \\
& =\left\langle A \varphi, \frac{1}{2 i}(1-U) \varphi\right\rangle-\left\langle\varphi, \frac{1}{2 i}(1-U) A \varphi\right\rangle \\
& =\frac{i}{2}\{\langle A \varphi, U \varphi\rangle-\langle\varphi, U A \varphi\rangle\},
\end{aligned}
$$

which implies the first two statements of point (a). Then, a successive use of (2.1) and (2.3) leads to the following equalities in $\mathscr{B}\left(\mathscr{D}(H), \mathscr{D}(H)^{*}\right)$ :

$$
\begin{aligned}
{[i H, A] } & =i(H+i)\left[A,(H+i)^{-1}\right](H+i) \\
& =2(1-U)^{-1}[A, U](1-U)^{-1} \\
& =-2\left(1-U^{*}\right)^{-1} U^{*}[A, U](1-U)^{-1} \\
& =-2\left\{(1-U)^{-1}\right\}^{*} U^{*}[A, U](1-U)^{-1},
\end{aligned}
$$

which imply the last statement of point (a). Now, relation (2.3) implies that

$$
\begin{gathered}
\int_{0}^{1} \frac{\mathrm{d} t}{t^{2}}\left\|\mathrm{e}^{-i t A}(H+i)^{-1} \mathrm{e}^{i t A}+\mathrm{e}^{i t A}(H+i)^{-1} \mathrm{e}^{-i t A}-2(H+i)^{-1}\right\| \\
=\frac{1}{2} \int_{0}^{1} \frac{\mathrm{d} t}{t^{2}}\left\|\mathrm{e}^{-i t A} U \mathrm{e}^{i t A}+\mathrm{e}^{i t A} U \mathrm{e}^{-i t A}-2 U\right\| .
\end{gathered}
$$


Thus, $H$ is of class $C^{1,1}(A)$ if and only if $U \in C^{1,1}(A)$. Since the same idea applies for the regularity condition $C^{1+0}(A)$, this proves point (b).

Remark 2.2. Due to the relation (2.3) between $U$ and $(H+i)^{-1}$, any regularity property of $U$ with respect to $A$ is equivalent to the same regularity property of $(H+i)^{-1}$ with respect to $A$. In particular, if $U$ belongs to one regularity class introduced in [3], Chapter 5, then the resolvent $(H+i)^{-1}$ belongs to the same regularity class. This observation might be useful in applications.

2.3. Mourre estimate. We start with a reformulation of Theorem 5.1 of [4] in the notations of Section 2.1. The proof is inspired from the corresponding proof in the self-adjoint case [3], Proposition 7.2.10.

Proposition 2.3 (Virial Theorem for $U$ ). Let $U$ and $A$ be respectively a unitary and a self-adjoint operator in $\mathscr{H}$, with $U \in C^{1}(A)$. Then,

$$
E^{U}(\{\theta\}) U^{*}[A, U] E^{U}(\{\theta\})=0
$$

for each $\theta \in \mathbb{T}$. In particular, one has

$$
\left\langle\varphi, U^{*}[A, U] \varphi\right\rangle=0
$$

for each eigenvector $\varphi$ of $U$.

Proof. One has to show that if $\varphi_{j} \in \mathscr{H}$ satisfies $U \varphi_{j}=\theta \varphi_{j}$ for $j=1,2$, then $\left\langle\varphi_{1}, U^{*}[A, U] \varphi_{2}\right\rangle=0$. But, since $U^{*} \varphi_{1}=\bar{\theta} \varphi_{1}$, this follows from the equalities

$$
\begin{aligned}
\left\langle\varphi_{1}, U^{*}[A, U] \varphi_{2}\right\rangle & =\lim _{\tau \rightarrow 0}\left\langle U \varphi_{1},\left[A_{\tau}, U\right] \varphi_{2}\right\rangle \\
& =\lim _{\tau \rightarrow 0}\left\{\left\langle\theta \varphi_{1}, A_{\tau} \theta \varphi_{2}\right\rangle-\left\langle\theta \bar{\theta} \varphi_{1}, A_{\tau} \varphi_{2}\right\rangle\right\} \\
& =0 .
\end{aligned}
$$

Corollary 2.4 (Discrete spectrum of $U$ ). Let $U$ and $A$ be respectively a unitary and a self-adjoint operator in $\mathscr{H}$, with $U \in C^{1}(A)$. Suppose there exist a Borel set $\Theta \subset \mathbb{T}$, a number $a>0$ and an operator $K \in \mathcal{K}(\mathscr{H})$ such that

$$
E^{U}(\Theta) U^{*}[A, U] E^{U}(\Theta) \geq a E^{U}(\Theta)+K .
$$

Then, the operator $U$ has at most finitely many eigenvalues in $\Theta$, each one of finite multiplicity.

The following proof is standard but we provide it for completeness. 
Proof. Let $\varphi \in \mathscr{H},\|\varphi\|=1$, be an eigenvector of $U$ with corresponding eigenvalue in $\Theta$. Then, it follows from (2.6) and from the Virial theorem that $\langle\varphi, K \varphi\rangle \leq-a$. Now, assume that the statement of the corollary is false. Then, there exists an infinite orthonormal sequence $\left\{\varphi_{j}\right\}$ of eigenvectors of $U$ in $E^{U}(\Theta) \mathscr{H}$. In particular, one has $\varphi_{j} \rightarrow 0$ weakly in $\mathscr{H}$ as $j \rightarrow \infty$. And since $K$ is compact, then $\left\langle\varphi_{j}, K \varphi_{j}\right\rangle \rightarrow 0$ as $j \rightarrow \infty$, which contradicts the inequality $\left\langle\varphi_{j}, K \varphi_{j}\right\rangle \leq-a<0$.

When a unitary operator $U$ satisfies an inequality (2.6), we say that a Mourre estimate for $U$ holds on $\Theta$. In the next proposition, we show that a Mourre estimate for $U$ implies a Mourre estimate for its Cayley transform $H \equiv i(1+U)(1-U)^{-1}$. We recall that for any bounded Borel set $I \subset \mathbb{R}$, the operator $E^{H}(I)$ belongs to $\mathscr{B}(\mathscr{H}, \mathscr{D}(H))$ and extends by duality to an element of $\mathscr{B}\left(\mathscr{D}(H)^{*}, \mathscr{H}\right)$.

Proposition 2.5 (Mourre estimate for $H$ ). Let $U$ and $A$ be respectively a unitary and a self-adjoint operator in $\mathcal{H}$, with $1 \notin \sigma_{\mathrm{p}}(U)$ and $U \in C^{1}(A)$. Suppose there exist a Borel set $\Theta \subset \mathbb{T}$, a number $a>0$ and an operator $K \in \mathcal{K}(\mathscr{H})$ such that

$$
E^{U}(\Theta) U^{*}[A, U] E^{U}(\Theta) \geq a E^{U}(\Theta)+K .
$$

Then, the operator $H$ defined by (2.2) is of class $C^{1}(A)$, and for any bounded Borel set $I \subset\left\{i \frac{1+\theta}{1-\theta} \mid \theta \in \Theta\right\}$ there exists an operator $K^{\prime} \in \mathcal{K}(\mathcal{H})$ such that

$$
E^{H}(I)[i A, H] E^{H}(I) \geq \frac{a}{2} E^{H}(I)+K^{\prime} .
$$

Proof. Lemma 2.1(a) implies that $H$ is of class $C^{1}(A)$ and that

$$
E^{H}(I)[i A, H] E^{H}(I)=2 E^{H}(I)\left\{(1-U)^{-1}\right\}^{*} U^{*}[A, U](1-U)^{-1} E^{H}(I) .
$$

Since $(H-z)^{-1}$ and $U$ commute for each $z \in \mathbb{C} \backslash \mathbb{R}$, one has

$$
(1-U)^{-1} E^{H}(I)=E^{H}(I)(1-U)^{-1} E^{H}(I) .
$$

So, this relation together with its adjoint imply that

$$
\begin{aligned}
& E^{H}(I) {[i A, H] E^{H}(I) } \\
&=2 E^{H}(I)\left\{(1-U)^{-1}\right\}^{*} E^{H}(I) U^{*}[A, U] E^{H}(I)(1-U)^{-1} E^{H}(I) .
\end{aligned}
$$

Since $E^{H}(I)=E^{U}(\Theta) E^{H}(I)$, it follows from (2.7) that

$$
E^{H}(I)[i A, H] E^{H}(I) \geq 2 a E^{H}(I)\left\{(1-U)^{-1}\right\}^{*}(1-U)^{-1} E^{H}(I)+K^{\prime}
$$

with $K^{\prime} \in \mathcal{K}(\mathscr{H})$. One concludes by noting that

$$
\left\{(1-U)^{-1}\right\}^{*}(1-U)^{-1}=\frac{1}{4}\left(H^{2}+1\right) \geq \frac{1}{4}
$$

holds on $E^{H}(I) \mathcal{H}$. 
We now prove a limiting absorption principle for the operator $H$ on the Besov space $\mathcal{E} \stackrel{\text { def }}{=}(\mathscr{D}(A), \mathcal{H})_{1 / 2,1}$ defined by real interpolation [3], Chapter 2 . We give two versions of the result: one if we know that $U$ has a spectral gap and another if we don't know it. We use the notation $\sigma_{\mathrm{p}}(H)$ for the point spectrum of $H$.

Proposition 2.6 (Limiting absorption principle for $H$ ). Let $U$ and $A$ be respectively a unitary and a self-adjoint operator in $\mathcal{H}$, with $1 \notin \sigma_{\mathrm{p}}(U)$. Assume either that $U$ has a spectral gap and $U \in C^{1,1}(A)$, or that $U \in C^{1+0}(A)$. Suppose also there exist a Borel set $\Theta \subset \mathbb{T}$, a number $a>0$ and an operator $K \in \mathcal{K}(\mathcal{H})$ such that

$$
E^{U}(\Theta) U^{*}[A, U] E^{U}(\Theta) \geq a E^{U}(\Theta)+K .
$$

Then, in any open bounded set $I \subset\left\{i \frac{1+\theta}{1-\theta} \mid \theta \in \Theta\right\}$ the operator $H$ has at most finitely many eigenvalues, each one of finite multiplicity. Furthermore, for each $\lambda \in I \backslash \sigma_{\mathrm{p}}(H)$ the limits $\lim _{\mu \searrow 0}(H-\lambda \mp i \mu)^{-1}$ exist in the weak $*$ topology of $\mathscr{B}\left(\mathscr{G}, \mathscr{G}^{*}\right)$, uniformly in $\lambda$ on each compact subset of $I \backslash \sigma_{\mathrm{p}}(H)$. As a corollary, $H$ has no singularly continuous spectrum in I.

If $U$ has a spectral gap, then so does $H$. In that case, the assumption $(H+i)^{-1} \in$ $C^{1,1}(A)$ (and accordingly the condition $U \in C^{1,1}(A)$ ) is optimal for the obtention of a limiting absorption principle for $H$ on the Besov scale $C^{s, p}(A)$ (see Appendix 7.B of [3]). The assumption $(H+i)^{-1} \in C^{1+0}(A)$ (and accordingly the condition $\left.U \in C^{1+0}(A)\right)$ is sufficient if $H$ has no gap, but it is slightly stronger than the $C^{1,1}$-condition. Another approach not requiring the existence of a gap exists and its regularity assumption is closer to the $C^{1,1}$-condition than the $C^{1+0}$-condition. However, its implementation is more involved since it requires the invariance of a certain domain under the group generated by $A$. So, we have decided not to present it for simplicity (see however [3], Section 7.5, for details).

Proof of Proposition 2.6. One first observes that $H$ has at most finitely many eigenvalues in $I$, each one of finite multiplicity. Indeed, this follows either from the corresponding statement for $U$ obtained in Corollary 2.4, or from the Mourre estimate (2.8) and [3], Corollary 7.2.11. Then, we know from [3], Lemma 7.2.12, that a strict Mourre estimate holds locally on $I \backslash \sigma_{\mathrm{p}}(H)$; that is, for any $\lambda \in I \backslash \sigma_{\mathrm{p}}(H)$ and any $\delta \in\left(0, \frac{a}{2}\right)$, there exists $\varepsilon>0$ such that

$$
E^{H}(\lambda ; \varepsilon)[i A, H] E^{H}(\lambda ; \varepsilon) \geq\left(\frac{a}{2}-\delta\right) E^{H}(\lambda ; \varepsilon),
$$

where

$$
E^{H}(\lambda ; \varepsilon) \stackrel{\text { def }}{=} E^{H}((\lambda-\varepsilon, \lambda+\varepsilon)) .
$$

Once this preliminary observation is made, the limiting absorption principle follows from Lemma 2.1(b) and [3], Theorem 7.4.1, in the case where $U$ has a spectral gap and $U \in C^{1,1}(A)$, while it follows from Lemma 2.1(b) and [22], Theorem 0.1, 
in the case where $U \in C^{1+0}(A)$. The fact $H$ that has no singularly continuous spectrum in $I$ is then a standard consequence of the limiting absorption principle and the finiteness of $\sigma_{\mathrm{p}}(H)$ in $I$.

2.4. Absolute continuity of $\boldsymbol{U}$. We are now in a position to prove our main result on the spectrum of $U$.

Theorem 2.7 (Spectral properties of $U$ ). Let $U$ and $A$ be respectively a unitary and a self-adjoint operator in $\mathscr{H}$. Assume either that $U$ has a spectral gap and $U \in C^{1,1}(A)$, or that $U \in C^{1+0}(A)$. Suppose also there exist an open set $\Theta \subset \mathbb{T}$, a number $a>0$ and an operator $K \in \mathcal{K}(\mathcal{H})$ such that

$$
E^{U}(\Theta) U^{*}[A, U] E^{U}(\Theta) \geq a E^{U}(\Theta)+K .
$$

Then, $U$ has at most finitely many eigenvalues in $\Theta$, each one of finite multiplicity, and $U$ has no singularly continuous spectrum in $\Theta$.

Proof. The properties of the eigenvalues of $U$ in $\Theta$ follow readily from Corollary 2.4. Now, choose a point $\omega \in \Theta \backslash \sigma_{\mathrm{p}}(U)$ and consider the unitary operator $U_{\omega} \stackrel{\text { def }}{=} \bar{\omega} U$. A direct verification shows that $U_{\omega}$ satisfies all the assumptions of Proposition 2.6 if $U$ is replaced by $U_{\omega}$ and $\Theta$ replaced by $\bar{\omega} \Theta$. Therefore, the Cayley transform $H_{\omega}$ of $U_{\omega}$ (see (2.2)) has no singularly continuous spectrum on any open bounded set $I \subset\left\{i \frac{1+\theta}{1-\theta} \mid \theta \in \bar{\omega} \Theta\right\}$. Using the correspondence between the spectral measures of $U_{\omega}$ and $H_{\omega}$ (see (2.4) and (2.5)), one infers that $U_{\omega}$ has no singularly continuous spectrum in $\bar{\omega} \Theta \backslash \mathcal{V}$, where $\mathcal{V}$ is any closed neighborhood of the point 1 . Since $\mathcal{V}$ is arbitrary, this implies that $U_{\omega}$ has no singularly continuous spectrum in $\bar{\omega} \Theta$, or equivalently that $U$ has no singularly continuous spectrum in $\Theta$.

Remark 2.8. If the Mourre estimate (2.9) holds with $K=0$, then the operator $U$ has only purely absolutely continuous spectrum in $\Theta$ (no point spectrum). Indeed, under this stronger assumption, inequality (2.8) is satisfied with $K^{\prime}=0$, which means that a strict Mourre estimate for $H$ holds locally. Then, the statement of Proposition 2.6 can be strengthened accordingly, and so does the statement of Theorem 2.7.

Our next goal is to exhibit locally $U$-smooth operators in our setting. Mimicking the corresponding definition in the self-adjoint case, we say that an operator $B \in$ $\mathscr{B}(\mathscr{H})$ is locally $U$-smooth on an open set $\Theta \subset \mathbb{T}$ if for each closed set $\Theta^{\prime} \subset \Theta$

$$
\sup _{\varphi \in \mathscr{H},\|\varphi\|=1} \sum_{n \in \mathbb{Z}}\left\|B U^{n} E^{U}\left(\Theta^{\prime}\right) \varphi\right\|^{2}<\infty .
$$

We also recall from [4], Theorem 2.2, that (2.10) is equivalent to

$$
\sup _{z \in \mathbb{D}, \varphi \in \mathscr{H},\|\varphi\|=1}\left|\left\langle\varphi, B \delta(U, z) E^{U}\left(\Theta^{\prime}\right) B^{*} \varphi\right\rangle\right|<\infty,
$$


with $\mathbb{D} \subset \mathbb{C}$ the open unit disk and

$$
\delta(U, z) \stackrel{\text { def }}{=}\left(1-z U^{*}\right)^{-1}-\left(1-\bar{z}^{-1} U^{*}\right)^{-1}
$$

the unitary version of the difference of resolvents. Note that if $\Theta^{\prime}=\mathbb{T}$ in (2.10), then $B$ is globally $U$-smooth in the usual sense [16], Section 7.

As in Section 2.2, we assume for a moment that $1 \notin \sigma_{\mathrm{p}}(U)$ and consider the Cayley transform $H$ of $U$. In this case, one has for $z \in \mathbb{C}$ with $|z| \neq 1$ that

$$
\left(1-z U^{*}\right)^{-1}=\left(1-z \frac{H+i}{H-i}\right)^{-1}=\frac{H-i}{1-z}\left(H-i \frac{1+z}{1-z}\right)^{-1},
$$

which implies that

$$
\begin{aligned}
\delta(U, z) & =\left(1-|z|^{2}\right)\left(1-z U^{*}\right)^{-1}\left\{\left(1-z U^{*}\right)^{-1}\right\}^{*} \\
& =\left(1-|z|^{2}\right) \frac{H-i}{1-z}\left(\frac{H-i}{1-z}\right)^{*}\left(H-i \frac{1+z}{1-z}\right)^{-1}\left\{\left(H-i \frac{1+z}{1-z}\right)^{-1}\right\}^{*} \\
& =\left(1-|z|^{2}\right) \frac{H^{2}+1}{|1-z|^{2}}\left(H-i \frac{1+z}{1-z}\right)^{-1}\left(H-i \frac{1+z}{1-z}\right)^{-1} .
\end{aligned}
$$

Moreover, let $\Theta^{\prime} \subset \mathbb{T} \backslash\{1\}$ be closed, and consider the compact set $I \subset \mathbb{R}$ given by

$$
I \stackrel{\text { def }}{=}\left\{i \frac{1+\theta}{1-\theta} \mid \theta \in \Theta^{\prime}\right\} .
$$

Since $E^{U}\left(\Theta^{\prime}\right)=E^{H}(I)$, it follows that

$$
H^{2} E^{U}\left(\Theta^{\prime}\right)=H^{2} E^{H}(I)=\eta(H) E^{H}(I)=\eta(H) E^{U}\left(\Theta^{\prime}\right)
$$

for some function $\eta \in C^{\infty}(\mathbb{R} ; \mathbb{R})$ with compact support. As a consequence, one infers that

$\delta(U, z) E^{U}\left(\Theta^{\prime}\right)=\left(1-|z|^{2}\right) \frac{\eta(H)+1}{|1-z|^{2}}\left(H-i \frac{1+z}{1-z}\right)^{-1}\left(H-i \overline{\frac{1+z}{1-z}}\right)^{-1} E^{U}\left(\Theta^{\prime}\right)$,

where $\eta(H) \in C^{1}(A)$ if $H$ is of class $C^{1}(A)$ (see [3], Theorem 6.2.5). Under the same regularity assumption on $H$, let us also observe that for any $s \in[0,1)$ the operator

$$
\langle A\rangle^{-s}(\eta(H)+1)\langle A\rangle^{s} \quad \text { with }\langle A\rangle \stackrel{\text { def }}{=} \sqrt{1+A^{2}},
$$

defined on $\mathscr{D}\left(\langle A\rangle^{S}\right)$, extends continuously to an element of $\mathscr{B}(\mathscr{H})$ (see [3], Proposition 5.3.1). With these preparations done, we can prove the existence of a large class of locally $U$-smooth operators.

Proposition 2.9 (Locally $U$-smooth operators). Let $U$ and $A$ be respectively a unitary and a self-adjoint operator in $\mathscr{H}$. Assume either that $U$ has a spectral gap and 
$U \in C^{1,1}(A)$, or that $U \in C^{1+0}(A)$. Suppose also there exist an open set $\Theta \subset \mathbb{\mathbb { T }}$, a number $a>0$ and an operator $K \in \mathcal{K}(\mathscr{H})$ such that

$$
E^{U}(\Theta) U^{*}[A, U] E^{U}(\Theta) \geq a E^{U}(\Theta)+K .
$$

Then, each operator $B \in \mathscr{B}(\mathcal{H})$ which extends continuously to an element of $\mathscr{B}\left(\mathscr{D}\left(\langle A\rangle^{S}\right)^{*}, \mathscr{H}\right)$ for some $s>1 / 2$ is locally $U$-smooth on $\Theta \backslash \sigma_{\mathrm{p}}(U)$.

Proof. It is sufficient to show that condition (2.11) holds for each closed set $\Theta^{\prime} \subsetneq$ $\Theta \backslash \sigma_{\mathrm{p}}(U)$. For the proof, we can assume without loss of generality that $B=\langle A\rangle^{-s}$ for some $s \in(1 / 2,1)$. We can also assume that $1 \notin\left\{\sigma_{\mathrm{p}}(U) \cup \Theta^{\prime}\right\}$. Indeed, if $1 \in\left\{\sigma_{\mathrm{p}}(U) \cup \Theta^{\prime}\right\}$, we can choose a point $\omega \in \Theta \backslash\left\{\sigma_{\mathrm{p}}(U) \cup \Theta^{\prime}\right\}$ (see Theorem

2.7) and replace the operator $U$ by the operator $U_{\omega} \stackrel{\text { def }}{=} \bar{\omega} U$, which now satisfies $1 \notin\left\{\sigma_{\mathrm{p}}\left(U_{\omega}\right) \cup \Theta^{\prime}\right\}$. Then, we can prove condition (2.11) for the operator $U_{\omega}$ on the set $\bar{\omega} \Theta^{\prime}$, and note that this is equivalent to the initial condition (2.11) since

$$
\begin{aligned}
& \sup _{z \in \mathbb{D}, \varphi \in \mathscr{H},\|\varphi\|=1}\left|\left\langle\varphi, B \delta\left(U_{\omega}, z\right) E^{U_{\omega}}\left(\bar{\omega} \Theta^{\prime}\right) B^{*} \varphi\right\rangle\right| \\
& =\sup _{z \in \mathbb{D}, \varphi \in \mathscr{H},\|\varphi\|=1}\left|\left\langle\varphi, B \delta(U, z) E^{U}\left(\Theta^{\prime}\right) B^{*} \varphi\right\rangle\right| .
\end{aligned}
$$

So, let's assume that $1 \notin\left\{\sigma_{\mathrm{p}}(U) \cup \Theta^{\prime}\right\}$. For $\varepsilon>0$, we consider the open set $\Theta_{\varepsilon}^{\prime} \subset \mathbb{D}$ given by

$$
\Theta_{\varepsilon}^{\prime} \stackrel{\text { def }}{=}\left\{z \in \mathbb{D}|| \Theta^{\prime}-z \mid<\varepsilon\right\} .
$$

By choosing $\varepsilon$ small enough, we can impose that the closure $\operatorname{cl}\left(\Theta_{\varepsilon}^{\prime}\right)$ of $\Theta_{\varepsilon}^{\prime}$ satisfies

$$
\Theta^{\prime} \subset \operatorname{cl}\left(\Theta_{\varepsilon}^{\prime}\right) \cap \mathbb{T} \subset \Theta \backslash\left(\sigma_{\mathrm{p}}(U) \cup\{1\}\right) .
$$

Then, one infers from the definition of $\delta(U, z)$ that

$$
\sup _{z \in \mathbb{D} \backslash \Theta_{\varepsilon}^{\prime}}\left\|\delta(U, z) E^{U}\left(\Theta^{\prime}\right)\right\|<\infty .
$$

Therefore, we can restrict our attention to the case $z \in \Theta_{\varepsilon}^{\prime}$ in condition (2.11). But, we know from (2.12) that

$$
\begin{aligned}
& \langle A\rangle^{-s} \delta(U, z) E^{U}\left(\Theta^{\prime}\right)\langle A\rangle^{-s} \\
& =\langle A\rangle^{-s}(\eta(H)+1)\langle A\rangle^{s}\left\{\frac{1-|z|^{2}}{|1-z|^{2}}\langle A\rangle^{-s}\left(H-i \frac{1+z}{1-z}\right)^{-1}\right. \\
& \left.\left(H-i \overline{\frac{1+z}{1-z}}\right)^{-1} E^{U}\left(\Theta^{\prime}\right)\langle A\rangle^{-s}\right\}
\end{aligned}
$$

for some $\eta \in C^{\infty}(\mathbb{R} ; \mathbb{R})$ with compact support. We know that $\langle A\rangle^{-s}(\eta(H)+1)\langle A\rangle^{s}$ defined on $\mathscr{D}\left(\langle A\rangle^{S}\right)$ extends continuously to an element of $\mathscr{B}(\mathscr{H})$. So, in order to 
control the norm of the operator in (2.14), we can further restrict our attention to the operator within the curly brackets for $z \in \Theta_{\varepsilon}^{\prime}$.

Using the notations

$$
\mu \stackrel{\text { def }}{=} \operatorname{Im}\left(i \frac{1+z}{1-z}\right) \equiv \frac{1-|z|^{2}}{|1-z|^{2}}
$$

and

$$
\lambda \stackrel{\text { def }}{=} \operatorname{Re}\left(i \frac{1+z}{1-z}\right) \equiv \frac{2 \operatorname{Im}(\bar{z})}{|1-z|^{2}},
$$

one gets the following bound for the norm the operator within the curly brackets:

$$
\begin{aligned}
& \left\|\mu\langle A\rangle^{-s}(H-\lambda-i \mu)^{-1}(H-\lambda+i \mu)^{-1} E^{U}\left(\Theta^{\prime}\right)\langle A\rangle^{-s}\right\| \\
& \quad \leq|\mu|\left\|\langle A\rangle^{-s}(H-\lambda-i \mu)^{-1}\right\|^{2} .
\end{aligned}
$$

Now, the set $\mathcal{V} \stackrel{\text { def }}{=}\left\{i \frac{1+z}{1-z} \mid z \in \mathrm{cl}\left(\Theta_{\varepsilon}^{\prime}\right) \cap \mathbb{T}\right\}$ is one of the compact subsets of $\mathbb{R} \backslash \sigma_{\mathrm{p}}(H)$ mentioned in Proposition 2.6. Hence, one infers from Proposition 2.6 that

$$
\begin{aligned}
\sup _{z \in \Theta_{\varepsilon}^{\prime}}|\mu|\left\|\langle A\rangle^{-s}(H-\lambda-i \mu)^{-1}\right\|^{2} & =\sup _{z \in \Theta_{\varepsilon}^{\prime}}\left\|\operatorname{Im}\left\{\langle A\rangle^{-s}(H-\lambda-i \mu)^{-1}\langle A\rangle^{-s}\right\}\right\| \\
& \leq \sup _{z \in \Theta_{\varepsilon}^{\prime}}\left\|\langle A\rangle^{-s}\left(H-i \frac{1+z}{1-z}\right)^{-1}\langle A\rangle^{-s}\right\| \\
& <\infty .
\end{aligned}
$$

This bound, together with (2.13) and (2.15), implies the claim.

To close the section, we give a corollary on the perturbations of $U$ which is useful for applications. For shortness, we state the result in the $C^{1+0}(A)$ case.

Corollary 2.10 (Perturbations of $U$ ). Let $A$ be a self-adjoint operator in $\mathcal{H}$ and let $U$ and $V$ be unitary operators in $\mathscr{H}$, with $U, V \in C^{1+0}(A)$. Suppose there exist an open set $\Theta \subset \mathbb{T}$, a number $a>0$ and an operator $K \in \mathcal{K}(\mathcal{H})$ such that

$$
E^{U}(\Theta) U^{*}[A, U] E^{U}(\Theta) \geq a E^{U}(\Theta)+K .
$$

Assume also that $V-1$ belongs to $\mathcal{K}(\mathscr{H})$. Then, $V U$ has at most finitely many eigenvalues in any closed subset of $\Theta$, each one of finite multiplicity, and $V U$ has no singularly continuous spectrum in $\Theta$.

Note that since $U V=U(V U) U^{*}$, the operators $U V$ and $V U$ are unitarily equivalent and thus have the same spectral properties. Note also that the two conditions $V-1 \in \mathcal{K}(\mathscr{H})$ and $V \in C^{1+0}(A)$ imply that $[A, V] \in \mathcal{K}(\mathscr{H})$, as shown for example in the proof of [3], Theorem 7.2.9. 
Proof. The product $V U$ belongs to $C^{1+0}(A)$, since $U$ and $V$ belong to $C^{1+0}(A)$ (see [3], Proposition 5.2.3(b)). Furthermore, a direct computation using the inclusion $[A, V] \in \mathcal{K}(\mathscr{H})$ implies that

$$
(V U)^{*}[A, V U]-U^{*}[A, U] \in \mathcal{K}(\mathscr{H}) .
$$

Now, since $V U-U=(V-1) U$ is compact, it follows from an application of the Stone-Weierstrass theorem that $\eta(V U)-\eta(U)$ is compact for any $\eta \in C(\mathbb{T})$. Therefore, for any $\eta \in C(\mathbb{T} ; \mathbb{R})$ satisfying $\eta(U)=\eta(U) E^{U}(\Theta)$, one infers from (2.16) and (2.17) that

$$
\begin{aligned}
\eta(V U)(V U)^{*}[A, V U] \eta(V U) & =\eta(U) E^{U}(\Theta) U^{*}[A, U] E^{U}(\Theta) \eta(U)+K_{1} \\
& \geq a \eta^{2}(U)+K_{2} \\
& =a \eta^{2}(V U)+K_{3},
\end{aligned}
$$

with $K_{1}, K_{2}, K_{3} \in \mathcal{K}(\mathscr{H})$. Since for each open set $\Theta^{\prime} \subset \mathbb{\mathbb { T }}$ with closure in $\Theta$, one can find $\eta \in C(\mathbb{T} ; \mathbb{R})$ such that $\eta(U)=\eta(U) E^{U}(\Theta)$ and $E^{V U}\left(\Theta^{\prime}\right)=$ $E^{V U}\left(\Theta^{\prime}\right) \eta(V U)$, it follows that

$$
\begin{aligned}
E^{V U} & \left(\Theta^{\prime}\right)(V U)^{*}[A, V U] E^{V U}\left(\Theta^{\prime}\right) \\
& =E^{V U}\left(\Theta^{\prime}\right) \eta(V U)(V U)^{*}[A, V U] \eta(V U) E^{V U}\left(\Theta^{\prime}\right) \\
& \geq a E^{V U}\left(\Theta^{\prime}\right)+K_{4},
\end{aligned}
$$

with $K_{4} \in \mathcal{K}(\mathscr{H})$. Thus, the claims for $V U$ follow directly from Theorem 2.7.

Example 2.11. A typical choice for the perturbation $V$ in Corollary 2.10 is $V=\mathrm{e}^{i B}$ with $B=B^{*} \in \mathcal{K}(\mathscr{H})$ satisfying $B \in C^{1+0}(A)$. Indeed, in such case we know from [3], Proposition 5.1.5, that $B^{k} \in C^{1}(A)$ and that

$$
\left[A, B^{k}\right]=\sum_{\ell=0}^{k-1} B^{k-1-\ell}[A, B] B^{\ell}
$$

for each $k \in \mathbb{N}^{*}$. Therefore, one has for each $\varphi \in \mathscr{D}(A)$

$$
\begin{aligned}
\left\langle A \varphi, \mathrm{e}^{i B} \varphi\right\rangle-\left\langle\varphi, \mathrm{e}^{i B} A \varphi\right\rangle & =\sum_{k \geq 1} \frac{i^{k}}{k !}\left(\left\langle A \varphi, B^{k} \varphi\right\rangle-\left\langle\varphi, B^{k} A \varphi\right\rangle\right) \\
& =\left\langle\varphi, \sum_{k \geq 1} \frac{i^{k}}{k !} \sum_{\ell=0}^{k-1} B^{k-1-\ell}[A, B] B^{\ell} \varphi\right\rangle,
\end{aligned}
$$


where $\sum_{k \geq 1} \frac{i^{k}}{k !} \sum_{\ell=0}^{k-1} B^{k-1-\ell}[A, B] B^{\ell}$ is a norm convergent sum of compact operators. It follows that $\mathrm{e}^{i B} \in C^{1}(A)$ with

$$
\left[A, \mathrm{e}^{i B}\right]=\sum_{k \geq 1} \frac{i^{k}}{k !} \sum_{\ell=0}^{k-1} B^{k-1-\ell}[A, B] B^{\ell} \in \mathcal{K}(\mathcal{H}) .
$$

Then, a simple calculation using the expression for $\left[A, \mathrm{e}^{i B}\right]$, the inclusion $B \in$ $C^{1+0}(A)$ and the fact that $C^{1+0}(A)$ is a vector space, shows that $\mathrm{e}^{i B} \in C^{1+0}(A)$. Since one also has $\mathrm{e}^{i B}-1 \in \mathcal{K}(\mathscr{H})$, all the assumptions on $V$ of Corollary 2.10 are satisfied.

\section{Applications}

3.1. Perturbations of bilateral shifts. Consider a unitary operator $U$ in a Hilbert space $\mathscr{H}$ and a subspace $\mathcal{M} \subset \mathscr{H}$ such that

$$
\mathcal{M} \perp U^{n}(\mathcal{M}) \text { for each } n \in \mathbb{Z} \backslash\{0\} \quad \text { and } \quad \mathscr{H}=\bigoplus_{n \in \mathbb{Z}} U^{n}(\mathcal{M}) .
$$

Such a unitary operator $U$, called a bilateral shift on $\mathscr{H}$ with wandering subspace $\mathcal{M}$, appears in various instances as in F. and M. Riesz theorem or in ergodic theory (see [21], Section 3). Using the notation $\varphi \equiv\left\{\varphi_{n}\right\}$ for elements of $\mathscr{H}$, we define the (number) operator

$$
A \varphi \stackrel{\text { def }}{=}\left\{n \varphi_{n}\right\}, \quad \varphi \in \mathscr{D}(A) \stackrel{\text { def }}{=}\left\{\psi \in \mathscr{H} \mid \sum_{n \in \mathbb{Z}} n^{2}\left\|\psi_{n}\right\|^{2}<\infty\right\} .
$$

The operator $A$ is self-adjoint since it can be regarded as a maximal multiplication operator acting in a $\ell^{2}$-space. Furthermore, a direct calculation shows that $\langle A \varphi, U \varphi\rangle-$ $\langle\varphi, U A \varphi\rangle=\langle\varphi, U \varphi\rangle$ for each $\varphi \in \mathscr{D}(A)$, meaning that $U \in C^{2}(A) \subset C^{1+0}(A)$ and that $U^{*}[A, U]=U^{*} U=1$.

Therefore, we infer from Theorem 2.7 and Remark 2.8 that $U$ has purely absolutely continuous spectrum, as it is well known. Now, let $V$ be another unitary operator with $V \in C^{1+0}(A)$ and $V-1 \in \mathcal{K}(\mathscr{H})$. Then, we deduce from Corollary 2.10 that the operator $V U$ has purely absolutely continuous spectrum except at a possible

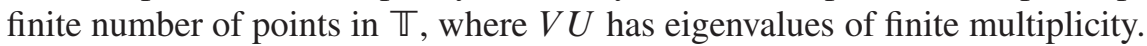

This result generalizes at the same time the results of Section 3 of [21] and Section 4.1 of [4] (in Theorem 4.1 of [4], a possible wandering subspace of the Hilbert space $L^{2}\left(\mathbb{R}^{m}\right)$ is the set of its elements having support in $\left\{x+t y \in \mathbb{R}^{m} \mid\right.$ $x \cdot y=0, t \in[0, T]\})$.

3.2. Perturbations of the Schrödinger free evolution. In this subsection we present an extension of Theorem 4.2 of [4]. So, for any $m \in \mathbb{N}^{*}$ let $\mathscr{H} \stackrel{\text { def }}{=} L^{2}\left(\mathbb{R}^{m}\right)$ and let

$$
Q \stackrel{\text { def }}{=}\left(Q_{1}, \ldots, Q_{m}\right) \text { and } P \stackrel{\text { def }}{=}\left(P_{1}, \ldots, P_{m}\right)
$$


be the usual families of momentum and position operators defined by

$$
\left(Q_{j} \varphi\right)(x) \stackrel{\text { def }}{=} x_{j} \varphi(x) \text { and }\left(P_{j} \varphi\right)(x) \stackrel{\text { def }}{=}-i\left(\partial_{j} \varphi\right)(x)
$$

for any Schwartz function $\varphi \in S\left(\mathbb{R}^{m}\right)$ and $x \in \mathbb{R}^{m}$. Our aim is to treat perturbations of the Schrödinger free evolution $U \stackrel{\text { def }}{=} \mathrm{e}^{-i T P^{2}}$, which is known to have purely absolutely continuous spectrum $\sigma(U)=\sigma_{\mathrm{ac}}(U)=\mathbb{T}$ for each $T>0$. Following [29], Definition 1.1, we consider the operator

$$
A \varphi \stackrel{\text { def }}{=} \frac{1}{2}\left\{\left(P^{2}+1\right)^{-1} P \cdot Q+Q \cdot P\left(P^{2}+1\right)^{-1}\right\} \varphi, \quad \varphi \in S\left(\mathbb{R}^{m}\right),
$$

which is essentially self-adjoint due to [3], Proposition 7.6.3(a). Then, a direct calculation shows that $\langle A \varphi, U \varphi\rangle-\langle\varphi, U A \varphi\rangle=\left\langle\varphi, 2 T U P^{2}\left(P^{2}+1\right)^{-1} \varphi\right\rangle$ for each $\varphi \in S\left(\mathbb{R}^{m}\right)$, meaning that $[A, U]=2 T U P^{2}\left(P^{2}+1\right)^{-1}$ by the density of $S\left(\mathbb{R}^{m}\right)$ in $\mathcal{D}(A)$. Therefore, one has $U \in C^{1}(A)$ with $U^{*}[A, U]=2 T P^{2}\left(P^{2}+1\right)^{-1}$, and further computations on $\delta\left(\mathbb{R}^{m}\right)$ show that $U \in C^{2}(A) \subset C^{1+0}(A)$. Now, observe that for any open set $\Theta \subset \mathbb{\mathbb { V }}$ with closure $\operatorname{cl}(\Theta)$ satisfying $\operatorname{cl}(\Theta) \cap\{1\}=\varnothing$, there exists $\delta>0$ such that

$$
E^{U}(\Theta)=E^{P^{2}}([\delta, \infty)) E^{U}(\Theta)
$$

and

$$
\begin{aligned}
E^{U}(\Theta) U^{*}[A, U] E^{U}(\Theta) & =2 T E^{U}(\Theta) P^{2}\left(P^{2}+1\right)^{-1} E^{P^{2}}([\delta, \infty)) E^{U}(\Theta) \\
& \geq 2 T \delta(\delta+1)^{-1} E^{U}(\Theta) .
\end{aligned}
$$

By combining what precedes together with Corollary 2.10, we obtain the following result.

Lemma 3.1. Let $V$ be a unitary operator in $\mathscr{H}$ satisfying $V \in C^{1+0}(A)$ and $V-1 \in$ $\mathcal{K}(\mathcal{H})$. Then, the eigenvalues of the operator $V U$ outside $\{1\}$ are of finite multiplicity and can accumulate only at $\{1\}$. Furthermore, VU has no singularly continuous spectrum.

3.3. Cocycles over irrational rotations. Consider two unitary operators $U$ and $V$ in a Hilbert space $\mathscr{H}$ satisfying, for some irrational $\theta \in[0,1)$, the commutation relation

$$
U V=\mathrm{e}^{2 \pi i \theta} V U
$$

The universal $C^{*}$-algebra $A_{\theta}$ generated by such a pair of unitary operators is known as the irrational rotation algebra [25], Section 12.3, and has attracted a lot of attention these last years. It is known that both $U$ and $V$ have full spectrum, that is,

$$
\sigma(U)=\sigma(V)=\mathbb{T}
$$


Our aim is to study the nature of the spectrum of $U$ when the pair $(U, V)$ is the following. Let $\mathscr{H} \stackrel{\text { def }}{=} \mathrm{L}^{2}([0,1))$ be the $\mathrm{L}^{2}$-space over the interval $[0,1)$ with addition modulo 1 , and let $V \in \mathscr{B}(\mathscr{H})$ be the unitary operator of multiplication by the independent variable, i.e.

$$
(V \varphi)(x) \stackrel{\text { def }}{=} \mathrm{e}^{2 \pi i x} \varphi(x) \text { for all } \varphi \in \mathscr{H} \text { and a.e. } x \in[0,1) .
$$

Given a measurable function $f:[0,1) \rightarrow \mathbb{R}$, let $U \in \mathcal{B}(\mathscr{H})$ be the unitary operator associated with the cocycle $f$ over the rotation by $\theta$, i.e.

$$
(U \varphi)(x) \stackrel{\text { def }}{=} \mathrm{e}^{2 \pi i f(x)} \varphi([x+\theta]) \quad \text { for all } \varphi \in \mathscr{H} \text { and a.e. } x \in[0,1),
$$

where $[y] \stackrel{\text { def }}{=} y$ modulo 1 . Then, $U$ and $V$ satisfy the commutation relation (3.1), and the spectrum of $U$ is of uniform multiplicity and either purely punctual, purely singularly continuous, or purely Lebesgue; see [11], Theorems 3 and 5. Note that the precise nature of the spectrum highly depends on properties of the pair $(f, \theta)$, see for example [14], [15], and [17].

In the sequel, we treat the case $f \stackrel{\text { def }}{=} m$ id $+h$, with $m \in \mathbb{Z}^{*}, \mathrm{id}:[0,1) \rightarrow[0,1)$ the identity function and $h:[0,1) \rightarrow \mathbb{R}$ an absolutely continuous function satisfying $h(0)=h(1)$. For this, we introduce the self-adjoint operator $P$ in $\mathscr{H}$ defined by

$$
P \varphi \stackrel{\text { def }}{=}-i \varphi^{\prime},
$$

$$
\varphi \in \mathscr{D}(P) \stackrel{\text { def }}{=}\left\{\varphi \in \mathscr{H} \mid \varphi \text { is absolutely continuous, } \varphi^{\prime} \in \mathscr{H} \text { and } \varphi(0)=\varphi(1)\right\} .
$$

Then, we observe that an integration by parts gives for each $\varphi \in \mathscr{D}(P)$ that

$$
\langle P \varphi, U \varphi\rangle-\langle\varphi, U P \varphi\rangle=\left\langle\varphi, 2 \pi\left(m+h^{\prime}\right) U \varphi\right\rangle,
$$

with $h^{\prime}$ the operator of multiplication by $h^{\prime}$. Therefore, if $h^{\prime} \in \mathrm{L}^{\infty}([0,1))$, one infers that $U \in C^{1}(P)$ with $U^{*}[P, U]=2 \pi\left(m+h^{\prime}([\cdot-\theta])\right)$. Moreover, by imposing some additional condition on the size of $h^{\prime}$, one would also obtain that $U^{*}[P, U]$ has a definite sign (the one of $m$ ). However, since this is not very satisfactory, we shall explain in the next proposition how this additional condition can be avoided by modifying adequately the conjugate operator $P$. The trick is based on the ergodic theorem and the new conjugate operator is of the form

$$
P_{n} \stackrel{\text { def }}{=} \frac{1}{n} \sum_{j=0}^{n-1} U^{-j} P U^{j}
$$

for some large $n \in \mathbb{N}^{*}$; see the appendix for abstract results on the self-adjointness of $P_{n}$ and on regularity properties with respect to $P_{n}$.

Proposition 3.2. Let $f \stackrel{\text { def }}{=} m$ id $+h$, with $m \in \mathbb{Z}^{*}$ and $h \in C^{1}([0,1) ; \mathbb{R})$ with $h^{\prime}$ Dini-continuous and $h(0)=h(1)$. Then the operator $U$, defined by (3.2) with $\theta$ irrational, has a purely Lebesgue spectrum of uniform multiplicity. Furthermore, the operator $\langle P\rangle^{-s}$ is globally $U$-smooth for any $s>1 / 2$. 
Proof. In what follows, we assume without loss of generality that $m \in \mathbb{N}^{*}$ (if $m<0$, the same proof works with $P$ replaced by $-P$ ).

Since $h^{\prime}$ is Dini-continuous, $h^{\prime} \in \mathrm{L}^{\infty}([0,1))$ and $U \in C^{1}(P)$ as indicated before. Thus, one infers from the appendix (with $A=P$ ) that for each $n \in \mathbb{N}^{*}$ the operator $P_{n}$, defined in (3.3), is self-adjoint on $\mathscr{D}\left(P_{n}\right) \stackrel{\text { def }}{=} \mathscr{D}(P)$. Moreover, one deduces from Lemma 4.1(a) that $U$ belongs to $C^{1}\left(P_{n}\right)$ with

$$
\begin{aligned}
{\left[P_{n}, U\right] } & =\frac{1}{n} \sum_{j=0}^{n-1} U^{-j}[P, U] U^{j} \\
& =\frac{2 \pi}{n} \sum_{j=0}^{n-1} U^{-j}\left(m+h^{\prime}\right) U^{j+1} \\
& =2 \pi U\left(m+\frac{1}{n} \sum_{j=1}^{n} h^{\prime}([\cdot-j \theta])\right) .
\end{aligned}
$$

Thus, one has

$$
U^{*}\left[P_{n}, U\right]=2 \pi\left(m+\frac{1}{n} \sum_{j=1}^{n} h^{\prime}([\cdot-j \theta])\right) .
$$

Also, since $\int_{0}^{1} \mathrm{~d} x h^{\prime}(x)=0$, it follows from the unique ergodicity of the irrational translation by $\theta$ that $\left|\frac{1}{n} \sum_{j=1}^{n} h^{\prime}([\cdot-j \theta])\right|<1 / 2$ for $n$ large enough. So, one deduces that $U^{*}\left[P_{n}, U\right] \geq \pi$ if $n$ is large enough.

To check that $U \in C^{1+0}\left(P_{n}\right)$, we observe from Lemma 4.1(b) that it is sufficient to show the inclusion $U \in C^{1+0}(P)$. We also recall that $[P, U]=2 \pi\left(m+h^{\prime}\right) U$. Therefore, since $U$ satisfies the condition $\int_{0}^{1} \frac{\mathrm{d} t}{t}\left\|\mathrm{e}^{-i t P} U \mathrm{e}^{i t P}-U\right\|<\infty$, one is reduced to showing that

$$
\int_{0}^{1} \frac{\mathrm{d} t}{t}\left\|\mathrm{e}^{-i t P}\left(m+h^{\prime}\right) \mathrm{e}^{i t P}-\left(m+h^{\prime}\right)\right\|=\int_{0}^{1} \frac{\mathrm{d} t}{t}\left\|\mathrm{e}^{-i t P} h^{\prime} \mathrm{e}^{i t P}-h^{\prime}\right\|<\infty .
$$

However, this condition is readily verified since it corresponds to nothing else but the Dini-continuity of $h^{\prime}$. One then concludes by applying Theorem 2.7 and Proposition 2.9 , and by taking into account the equality $\mathscr{D}\left(P_{n}\right)=\mathscr{D}(P)$.

The first part of Proposition 3.2 is not new; the nature of the spectrum of $U$ was already determined in [14] and [15] under a slightly weaker assumption ( $h$ absolutely continuous with $h^{\prime}$ of bounded variation). On the other hand, we have not been able to find in the literature any information about globally $U$-smooth operators. Therefore, the second part Proposition 3.2 is apparently new. 
3.4. Vector fields on manifolds. Let $M$ be a smooth orientable manifold of dimension $n \geq 1$ with volume form $\Omega$, and let $\mathscr{H} \stackrel{\text { def }}{=} \mathrm{L}^{2}(M, \Omega)$ be the corresponding $\mathrm{L}^{2}$-space. Consider a complete $C^{\infty}$ vector field $X \in \mathfrak{X}(M)$ on $M$ with flow $\mathbb{R} \times M \ni(t, p) \mapsto F_{t}(p) \in M$. Then, there exists for each $t \in \mathbb{R}$ a unique function $\operatorname{det}_{\Omega}\left(F_{t}\right) \in C^{\infty}(M ; \mathbb{R})$ satisfying $F_{t}^{*} \Omega=\operatorname{det}_{\Omega}\left(F_{t}\right) \Omega$, with $F_{t}^{*}$ the pullback by $F_{t}\left(\operatorname{det}_{\Omega}\left(F_{t}\right)\right.$ is the determinant of $F_{t}$, see [1], Definition 2.5.18). Furthermore, the continuity of the map $t \mapsto F_{t}(\cdot)$, together with the fact that $F_{0}=\mathrm{id}_{M}$ is orientationpreserving, implies that $\operatorname{det}_{\Omega}\left(F_{t}\right)>0$ on $M$ for all $t \in \mathbb{R}$. So, one can define for each $t \in \mathbb{R}$ the operator

$$
U_{t} \varphi \stackrel{\text { def }}{=}\left\{\operatorname{det}_{\Omega}\left(F_{t}\right)\right\}^{1 / 2} F_{t}^{*} \varphi, \quad \varphi \in \mathscr{H},
$$

which is easily shown to be unitary in $\mathscr{H}$ (use for example [1], Proposition 2.5.20). Now, let

$$
M_{0} \stackrel{\text { def }}{=}\left\{p \in M \mid X_{p}=0\right\}
$$

be the set of critical points of $X$. Then, the subspaces $\mathrm{L}^{2}\left(M_{0}, \Omega\right)$ and $\mathrm{L}^{2}\left(M \backslash M_{0}, \Omega\right)$ reduce $U_{t}$, with $U_{t} \uparrow \mathrm{L}^{2}\left(M_{0}, \Omega\right)=1$ and $U_{t} \uparrow \mathrm{L}^{2}\left(M \backslash M_{0}, \Omega\right)$ unitary. Therefore, we can restrict our attention to the unitary operator $U_{t}^{0} \stackrel{\text { def }}{=} U_{t} \uparrow \mathrm{L}^{2}\left(M \backslash M_{0}, \Omega\right)$ in $\mathscr{H}_{0} \stackrel{\text { def }}{=} \mathrm{L}^{2}\left(M \backslash M_{0}, \Omega\right)$.

Suppose that there exist a function $g \in C^{1}\left(M \backslash M_{0}\right.$; $\left.\mathbb{R}\right)$ and a constant $\delta>0$ such that

$$
\mathrm{d} g \cdot X \geq \delta \quad \text { and } \quad \mathrm{d} g \cdot X \in \mathrm{L}^{\infty}\left(M \backslash M_{0}, \Omega\right) .
$$

Then, the multiplication operator

$$
A \varphi \stackrel{\text { def }}{=}-g \varphi, \quad \varphi \in C_{\mathrm{c}}\left(M \backslash M_{0}\right),
$$

is essentially self-adjoint in $\mathscr{H}_{0}$ [19], Example 5.1.15, and a direct calculation using the inclusion $U_{t}^{0} C_{\mathrm{c}}\left(M \backslash M_{0}\right) \subset C_{\mathrm{c}}\left(M \backslash M_{0}\right)$ implies for each $\varphi \in C_{\mathrm{c}}\left(M \backslash M_{0}\right)$ that

$$
\begin{aligned}
\left\langle A \varphi, U_{t}^{0} \varphi\right\rangle_{\mathscr{H}_{0}}-\left\langle\varphi, U_{t}^{0} A \varphi\right\rangle_{\mathscr{H}_{0}} & =\left\langle\varphi, U_{t}^{0}\left(U_{-t}^{0} A U_{t}^{0}-A\right) \varphi\right\rangle_{\mathscr{H}_{0}} \\
& =-\left\langle\varphi, U_{t}^{0}\left(F_{-t}^{*} g-g\right) \varphi\right\rangle_{\mathscr{H}_{0}} \\
& =\left\langle\varphi, U_{t}^{0} \int_{0}^{t} \mathrm{~d} s(\mathrm{~d} g)_{F_{-s}} \cdot X_{F_{-s}} \varphi\right\rangle_{\mathscr{H}_{0}},
\end{aligned}
$$

where $\int_{0}^{t} \mathrm{~d} s(\mathrm{~d} g)_{F_{-s}} \cdot X_{F_{-s}}$ is the multiplication operator by the function

$$
p \longmapsto \int_{0}^{t} \mathrm{~d} s(\mathrm{~d} g)_{F_{-s}(p)} \cdot X_{F_{-s}(p)} .
$$

This, together with the density of $C_{\mathrm{c}}\left(M \backslash M_{0}\right)$ in $\mathscr{D}(A)$ and the second condition of (3.4), implies that

$$
\left[A, U_{t}^{0}\right]=U_{t}^{0} \int_{0}^{t} \mathrm{~d} s(\mathrm{~d} g)_{F_{-s}} \cdot X_{F_{-s}} \in \mathscr{B}\left(\mathscr{H}_{0}\right)
$$


and that

$$
\left[A,\left[A, U_{t}^{0}\right]\right]=U_{t}^{0}\left(\int_{0}^{t} \mathrm{~d} s(\mathrm{~d} g)_{F_{-s}} \cdot X_{F_{-s}}\right)^{2} \in \mathscr{B}\left(\mathscr{H}_{0}\right) .
$$

So, $U_{t}^{0} \in C^{2}(A) \subset C^{1+0}(A)$, and the first condition of (3.4) gives for any $t>0$

$$
\left(U_{t}^{0}\right)^{*}\left[A, U_{t}^{0}\right]=\int_{0}^{t} \mathrm{~d} s(\mathrm{~d} g)_{F_{-s}} \cdot X_{F_{-s}} \geq \delta t .
$$

Therefore, we infer from Theorem 2.7 and Remark 2.8 that $U_{t}^{0}$ is purely absolutely continuous in $\mathscr{H}_{0}$ for any $t>0$ (and thus for all $t \neq 0$ since $\left.U_{-t}^{0}=\left(U_{t}^{0}\right)^{*}\right)$. In particular, $U_{t}$ is purely absolutely continuous in $\mathscr{H}$ for any $t \neq 0$ if the measure of $M_{0}$ (relative to $\Omega$ ) is zero. This result complements [20], Section 2.9(ii), where the author treats the case of unitary operators induced by divergence-free vector fields on connected open subsets of $\mathbb{R}^{n}$.

To conclude this subsection, we exhibit an explicit class of vector fields on $\mathbb{R}^{n}$ satisfying all of our assumptions. Take $M=\mathbb{R}^{n}$ and let $X \in \mathfrak{X}\left(\mathbb{R}^{n}\right)$ be given by

$$
X_{x} \stackrel{\text { def }}{=} f(|x|) \frac{x}{|x|}, \quad x \in \mathbb{R}^{n},
$$

where $f \in C^{\infty}\left([0, \infty)\right.$; R ) satisfies $\lim _{r \searrow 0}|f(r) / r|<\infty$ and $\delta_{1} r \leq f(r) \leq$ $\delta_{2} r+\delta_{3}$ for all $r \geq 0$ and some $\delta_{1}, \delta_{2}, \delta_{3}>0$. Then, we have $M_{0}=\{0\}$, and we know from [18], Remark 1.8.7, that $X$ is complete. Therefore, the restriction

$$
U_{t}^{0} \stackrel{\text { def }}{=} U_{t} \uparrow \mathrm{L}^{2}\left(\mathbb{R}^{n} \backslash\{0\}, \mathrm{d} x\right)
$$

is a well defined unitary operator. Now, let $g \in C^{1}\left(\mathbb{R}^{n} \backslash\{0\} ; \mathbb{R}\right)$ be given by $g(x) \stackrel{\text { def }}{=}$ $\ln \left(|x|^{2}\right)$. Then, the conditions (3.4) are verified due to the properties of the function $f$, and so $U_{t}^{0}$ is purely absolutely continuous in $\mathrm{L}^{2}\left(\mathbb{R}^{n} \backslash\{0\}, \mathrm{d} x\right)$ for any $t \neq 0$. Since $M_{0}$ has Lebesgue measure zero, it follows that $U_{t}$ is purely absolutely continuous in $\mathrm{L}^{2}\left(\mathbb{R}^{n}, \mathrm{~d} x\right)$. This covers for instance the well known case of the dilation group, where $f(r)=r$.

\section{Appendix}

In this appendix, we introduce an abstract class of conjugate operators which is useful for the study of cocycles in Section 3.3.

Let $A$ and $U$ be respectively a self-adjoint and a unitary operator in a Hilbert space $\mathscr{H}$. If $U \in C^{1}(A)$, then we know from [3], Propositions 5.1.5 and 5.1.6, that $U^{k} \in C^{1}(A)$ for each $k \in \mathbb{Z}$ and $U^{k} \mathscr{D}(A)=\mathscr{D}(A)$. Therefore, for each $n \in \mathbb{N}^{*}$ the operator $\frac{1}{n} \sum_{j=0}^{n-1} U^{-j}\left[A, U^{j}\right]$ is bounded, and the operator

$$
A_{n} \varphi \stackrel{\text { def }}{=} \frac{1}{n} \sum_{j=0}^{n-1} U^{-j} A U^{j} \varphi \equiv \frac{1}{n} \sum_{j=0}^{n-1} U^{-j}\left[A, U^{j}\right] \varphi+A \varphi, \quad \varphi \in \mathscr{D}\left(A_{n}\right) \stackrel{\text { def }}{=} \mathcal{D}(A),
$$


is self-adjoint. Furthermore, we have the following result on the regularity of $U$ with respect to $A_{n}$.

Lemma 4.1. Take $n \in \mathbb{N}^{*}$ and let $A$ and $U$ be respectively a self-adjoint and a unitary operator in a Hilbert space $\mathscr{H}$. Then,

(a) if $U \in C^{1}(A)$, then $U \in C^{1}\left(A_{n}\right)$ with $\left[A_{n}, U\right]=\frac{1}{n} \sum_{j=0}^{n-1} U^{-j}[A, U] U^{j}$;

(b) if $U \in C^{1+0}(A)$, then $U \in C^{1+0}\left(A_{n}\right)$.

Proof. (a) Since $U^{k} \in C^{1}(A)$ for each $k \in \mathbb{Z}$, one has for any $\varphi \in \mathscr{D}\left(A_{n}\right)$ that

$$
\begin{aligned}
\left\langle A_{n} \varphi, U \varphi\right\rangle-\left\langle\varphi, U A_{n} \varphi\right\rangle & =\frac{1}{n} \sum_{j=0}^{n-1}\left\langle\varphi,\left(U^{-j} A U^{j+1}-U^{1-j} A U^{j}\right) \varphi\right\rangle \\
& =\frac{1}{n} \sum_{j=0}^{n-1}\left\langle\varphi, U^{-j}[A, U] U^{j} \varphi\right\rangle,
\end{aligned}
$$

with $[A, U] \in \mathscr{B}(\mathcal{H})$. This implies the claim.

(b) We know from point (a) that $U \in C^{1}\left(A_{n}\right)$ with

$$
\left[A_{n}, U\right]=\frac{1}{n} \sum_{j=0}^{n-1} U^{-j}[A, U] U^{j} .
$$

Therefore, it is sufficient to show for each $j \in\{1, \ldots, n-1\}$ that

$$
\int_{0}^{1} \frac{\mathrm{d} t}{t}\left\|\mathrm{e}^{-i t A_{n}} B_{j} \mathrm{e}^{i t A_{n}}-B_{j}\right\|<\infty
$$

with $B_{j} \stackrel{\text { def }}{=} U^{-j}[A, U] U^{j}$. But, for each $t \in \mathbb{R}$ and each $\varphi \in \mathscr{D}(A)$ we have

$$
\begin{aligned}
\mathrm{e}^{i t A_{n}} \varphi-\mathrm{e}^{i t A} \varphi & =\int_{0}^{t} \mathrm{~d} s \frac{\mathrm{d}}{\mathrm{d} s}\left(\mathrm{e}^{i s A_{n}} \mathrm{e}^{-i s A}-1\right) \mathrm{e}^{i t A} \varphi \\
& =\int_{0}^{t} \mathrm{~d} s \frac{i}{n} \sum_{k=0}^{n-1} \mathrm{e}^{i s A_{n}} U^{-k}\left[A, U^{k}\right] \mathrm{e}^{i(t-s) A} \varphi .
\end{aligned}
$$

So, there exists $C_{t} \in \mathscr{B}(\mathscr{H})$ with $\left\|C_{t}\right\| \leq$ Const. $|t|$ such that $\mathrm{e}^{i t A_{n}}=\mathrm{e}^{i t A}+C_{t}$, and thus

$$
\int_{0}^{1} \frac{\mathrm{d} t}{t}\left\|\mathrm{e}^{-i t A_{n}} B_{j} \mathrm{e}^{i t A_{n}}-B_{j}\right\| \leq \text { Const }+\int_{0}^{1} \frac{\mathrm{d} t}{t}\left\|\mathrm{e}^{-i t A} B_{j} \mathrm{e}^{i t A}-B_{j}\right\| .
$$

Now, the integral $\int_{0}^{1} \frac{\mathrm{d} t}{t}\left\|\mathrm{e}^{-i t A} D \mathrm{e}^{i t A}-D\right\|$ is finite for $D=U^{-j}, D=[A, U]$ and $D=U^{j}$ due to the assumption. So, the integral in the right hand side of (4.1) is also finite, and thus the claim is proved. 


\section{References}

[1] R. Abraham and J. E. Marsden, Foundations of mechanics. Second edition. Benjamin/Cummings Publishing Co., Reading, MA, 1978. MR 0515141 Zbl 0393.70001

[2] W. O. Amrein, Hilbert space methods in quantum mechanics. Fundamental Sciences. EPFL Press, Lausanne, 2009. MR 2571750 Zbl 1207.46001

[3] W. O. Amrein, A. Boutet de Monvel, and V. Georgescu, $C_{0}$-groups, commutator methods and spectral theory of $N$-body Hamiltonians. Progress in Mathematics 135. Birkhäuser, Basel, 1996. MR 1388037 Zbl 0962.47500

[4] M. A. Astaburuaga, O. Bourget, V. H. Cortés, and C. Fernández, Floquet operators without singular continuous spectrum. J. Funct. Anal. 238 (2006), 489-517. MR 2253730 Zbl 1106.47034

[5] J. Bellissard, Noncommutative methods in semiclassical analysis. In S. Graffi (ed), Transition to chaos in classical and quantum mechanics. Lectures given at the $3^{\text {rd }}$ session of the Centro Internazionale Matematico Estivo (C.I.M.E.) held in Montecatini Terme, Italy, July 6-13, 1991. Lecture Notes in Mathematics 1589. Springer Verlag, Berlin, 1994, 1-64. MR 1323220 Zbl 0818.46072

[6] J. Blank, P. Exner, and M. Havlíček, Hilbert space operators in quantum physics. Second ed. Theoretical and Mathematical Physics. Springer Verlag, Berlin etc., and AIP Press, New York, 2008. MR 2458485 Zbl 1163.47060

[7] J. E. Fornæss and B. Weickert, A quantized Hénon map. Discrete Contin. Dynam. Systems 6 (2000), 723-740. MR 1757399 Zbl 1009.37052

[8] D. S. Gilliam, J. R. Schulenberger, and J. L. Lund, Spectral representation of the Laplace and Stieltjes transforms. Mat. Apl. Comput. 7 (1988), 101-107. MR 0987878 Zbl 0666.44001

[9] L. Golinskii, A. Kheifets, F. Peherstorfer, and P. Yuditskii, Scattering theory for CMV matrices: uniqueness, Helson-Szegö and strong Szegö theorems. Integral Equations Operator Theory 69 (2011), 479-508. MR 2784575 Zbl 1235.47029

[10] I. Guarneri, On the spectrum of the resonant quantum kicked rotor. Ann. Henri Poincaré 10 (2009), 1097-1110. MR 2557197 Zbl 1206.81049

[11] H. Helson, Cocycles on the circle. J. Operator Theory 16 (1986), 189-199. MR 0847339 Zbl 0644.43003

[12] M. J. Huang, On the absolutely continuous subspaces of Floquet operators. Proc. Roy. Soc. Edinburgh Sect. A 124 (1994), 703-712. MR 1298587 Zbl 0816.47033

[13] M. J. Huang and R. B. Lavine, Boundedness of kinetic energy for time-dependent Hamiltonians. Indiana Univ. Math. J. 38 (1989), 189-210. MR 0982577 Zbl 0672.47006

[14] A. Iwanik, M. Lemańczyk, and C. Mauduit, Piecewise absolutely continuous cocycles over irrational rotations. J. London Math. Soc. (2) 59 (1999), 171-187. MR 1688497 Zbl 0931.28015

[15] A. Iwanik, M. Lemańczyk, and D. Rudolph, Absolutely continuous cocycles over irrational rotations. Israel J. Math. 83 (1993): 73-95. MR 1239717 Zbl 0786.28011

[16] T. Kato, Smooth operators and commutators. Studia Math. 31 (1968), 535-546. MR 0234314 Zbl 0215.48802 
[17] H. A. Medina, Spectral types of unitary operators arising from irrational rotations on the circle group. Michigan Math. J. 41 (1994), 39-49. MR 1260607 Zbl 0999.47024

[18] R. Narasimhan, Analysis on real and complex manifolds. Second edition. Masson \& Cie, Éditeur, Paris, North-Holland Publishing Co., Amsterdam and London, and American Elsevier Publishing Co., New York, 1973. MR 0346855 Zbl 0188.25803

[19] G. K. Pedersen, Analysis now. Graduate Texts in Mathematics 118. Springer Verlag, New York, 1989. MR 0971256 Zbl 0668.46002

[20] C. R. Putnam, Commutation properties of Hilbert space operators and related topics. Ergebnisse der Mathematik und ihrer Grenzgebiete 36. Springer Verlag New York, New York, 1967. MR 0217618 Zbl 0149.35104

[21] C. R. Putnam, Absolute continuity and hyponormal operators. Internat. J. Math. Math. Sci. 4 (1981), 321-335. MR 0613253 Zbl 0463.47019

[22] J. Sahbani, The conjugate operator method for locally regular Hamiltonians. J. Operator Theory 38 (1997), 297-322. MR 1606944 Zbl 0905.47003

[23] B. Simon, CMV matrices: five years after. J. Comput. Appl. Math. 208 (2007), 120-154. MR 2347741 Zbl 1125.15027

[24] D. C. Szajda, Absolute continuity of a class of unitary operators. Houston J. Math. 27 (2001), 189-202. MR 1843918 Zbl 0988.47010

[25] N. E. Wegge-Olsen, $K$-theory and $C^{*}$-algebras, A friendly approach. Oxford Science Publications. The Clarendon Press, Oxford University Press, New York, 1993. MR $1222415 \mathrm{Zbl} 0780.46038$

[26] B. Weickert, Quantizations of linear self-maps of $\mathbb{R}^{2}$. Acta Sci. Math. (Szeged) 69 (20003), 619-631. MR 2034197 Zbl 1064.47033

[27] B. Weickert, Spectral properties and dynamics of quantized Henon maps. Trans. Amer. Math. Soc. 356 (2004), 4951-4968. MR 2084407 Zbl 1070.37028

[28] J. Weidmann, Linear operators in Hilbert spaces. Graduate Texts in Mathematics 68. Springer Verlag, New York etc., 1980. MR 0566954 Zbl 0434.47001

[29] K. Yokoyama, Mourre theory for time-periodic systems. Nagoya Math. J. 149 (1998), 193-210. MR 1619581 Zbl 0916.47038

Received April 14, 2012; revised June 27, 2012

Claudio Fernández, Facultad de Matemáticas, Pontificia Universidad Católica de Chile,

Av. Vicuña Mackenna 4860, Santiago, Chile

E-mail: cfernand@mat.puc.cl

Serge Richard, Université de Lyon; Université Lyon 1;

CNRS, UMR5208, Institut Camille Jordan, 43 blvd du 11 novembre 1918,

F-69622 Villeurbanne-Cedex, France

E-mail: richard@math.univ-lyon1.fr

Rafael Tiedra de Aldecoa, Facultad de Matemáticas,

Pontificia Universidad Católica de Chile, Av. Vicuña Mackenna 4860, Santiago, Chile

E-mail: rtiedra@mat.puc.cl 